\title{
Management of Hepatic Artery Thrombosis after Living Donor Liver Transplantation: Endovascular Thrombolytic Therapy or Surgical Intervention? Role of Hepatic Arterial Urokinase Infusion
}

\author{
Ashok Thorat ${ }^{1,2}$, Long-Bin Jeng ${ }^{1,2,3}$, Shih-Chao Hsur ${ }^{1,2,3}$, Ping-Chun Li ${ }^{1,2,4}$, Chun-Chieh Yeh ${ }^{1,2,3}$, Te-Hung Chen ${ }^{1,2,3}$, Horng-Ren Yang ${ }^{1,2,3}$, \\ Kin-Shing Poon ${ }^{2,5}$ \\ 'Organ Transplantation Centre, China Medical University Hospital, Taichung, Taiwan \\ ${ }^{2}$ China Medical University, Taichung, Taiwan \\ ${ }^{3}$ Department of Surgery, China Medical University Hospital, Taichung, Taiwan \\ ${ }^{4}$ Department of cardiovascular surgery, China Medical University Hospital, Taichung, Taiwan \\ ${ }^{5}$ Department of Anaesthesiology, China Medical University Hospital, Taichung, Taiwan \\ Corresponding author: \\ Long-Bin Jeng, MD \\ Organ Transplantation Center \\ China Medical University Hospital \\ 2, Yuh-Der Road, Taichung, Taiwan \\ 40447 \\ TEL: 04-22052121Ext.1765 \\ FAX: $04-22029083$ \\ E-mail: otc@mail.cmuh.org.tw
}

\section{ABSTRACT}

The hepatic artery (HA) related complications after living donor liver transplantation (LDLT) remains an important cause of increased risk of post-transplant mortality. The timing of occurrence of hepatic artery thrombosis (HAT) after transplantation and the timely intervention are the determining factors in the survival of the recipients. Re-exploration and revision of the hepatic artery anastomosis or medical treatment with urokinase therapy are the lifesaving treatment options. We describe our successful outcome after arterial urokinase infusion as an initial therapy for the LDLT recipients with HAT.

Materials and Methods: From $10^{\text {th }}$ September 2002 till $31^{\text {st }}$ December 2017, a total of 908 LDLT surgeries were performed at China Medical University Hospital. A total of 12 patients that developed HAT in postoperative period were further studied. All the patients that were diagnosed to have HAT within 24 hours were re-explored whereas medical therapy with intraarterial urokinase infusion was the first treatment modality for the HAT developing after 24 hours of LDLT.

Results: 12 recipients (1.37\%) developed HAT. Computed tomography (CT) angiography was done for all the recipients and arterial Urokinase infusion was given in 10 patients as the initial therapy whereas 2 patients were directly re-explored. All the urokinase treated patients had HAT 24 hours after LDLT. Five of the urokinase therapy patients developed complete re-canalization after urokinase therapy without any morbidity in post-transplant period. After successful re-canalization, there was no evidence of arterial stenosis in subsequent follow up with satisfactory liver graft functions. Remaining five patients underwent re-exploration with revision of hepatic artery anastomosis $(n=2)$ and re-transplantation $(n=3)$.

Conclusions: Intra-arterial urokinase therapy is a feasible initial therapy in LDLT recipients with HAT with an acceptable HA recanalization rate that needs no further surgical intervention and can certainly be first choice of treatment in stable HAT patients. However, failure of re-canalization or deterioration of the patient's clinical condition warrants urgent surgical intervention.

Key words: hepatic artery thrombosis, living donor liver transplantation, urokinase therapy, re-transplantation. 


\section{INTRODUCTION}

Hepatic arterial reconstruction remains a challenging issue in LDLT due to smaller diameter of the graft HA. The complications related to HA in post LDLT period such as HAT is associated with an increased risk of liver allograft loss if not treated promptly $(1,2)$. The timing of occurrence of HAT after transplantation and the timely intervention are the determining factors in the survival of the recipients. The HAT is reported to occur from $1.4 \%$ to 9.2\% after adult LDLT surgeries $(1,3)$. The mortality directly related to HAT is as high as $55.6 \%$ in early thrombosis compared with $15-22.6 \%$ because of late thrombosis (4). The typical course of HAT is graft ischemia and hepatic infarction, followed by septicemia and hence, urgent intervention is recommended. It requires early diagnosis and revascularization to avoid graft loss. Failure to achieve recanalization and establishment of the HA blood flow warrants urgent retransplantation.

Although, performing arterial reconstruction using microsurgical technique (5) or using surgical loupes (1) have lowered the incidence of HAT after LDLT, in most cases HAT that occurs early after liver transplantation is considered as a technical surgical complication. The most effective treatment modality in this scenario remains controversial. Vascular complications can be treated by several approaches: revascularization, re-transplantation, and endovascular management that depends upon the clinical presentation of HAT, as well as the clinical condition of the patient, which may vary considerably from benign to severe $(6,7)$. Revascularization can be achieved by surgical re-exploration and thrombectomy (with or without re-do of the arterial anastomosis), or percutaneous hepatic arterial thrombolysis $(1,8)$. Failure of arterial recanalization leads to irreversible liver graft damage and clinical deterioration of the patient that warrants urgent liver re-transplantation.

The HAT developing in immediate transplant period is best managed by emergency surgical revision of the anastomosis (1). Surgical revascularization, thus, is an effective alternative for graft salvage or may help as a bridging measure for a re-transplantation in a less emergent setting. In hemodynamically stable patients, however, less invasive treatment options such as endovascular thrombolysis can be tried. In recent era, hepatic arterial urokinase therapy has emerged as a successful treatment optionfor treating early HAT that can avoid need for surgical intervention and retransplantation. In this study, we aim to evaluate the feasibility of the endovascular approach and role of urokinase therapy for HAT developing after LDLT.

\section{MATERIALS AND METHODS}

From $10^{\text {th }}$ September 2002 till $31^{\text {st }}$ December 2017, a total of 908 LDLT surgeries were performed at China Medical University Hospital, Taiwan. Database of the patients with $\mathrm{HA}$ related complications was assessed and a total of 12 patients that developed HAT in postoperative period were further studied. The duration of the development of the HAT after liver transplantation was recorded. Early and late HAT were defined by the occurrence of arterial thrombosis within or later than $\mathbf{3 0}$ days after LDLT, respectively. Clinical presentation of the HAT, therapy administered, and the outcome of the patients were analyzed. The pretransplant medical records, operative details, time to develop HAT, and the treatment methods were recorded. All the patients that were diagnosed to have HAT within 24 hours were re-explored whereas medical therapy with intra-arterial urokinase infusion was the first treatment modality for the HAT developing after 24 hours of LDLT. Follow up period ranged from 3 months to 15 years.

\section{Technique of hepatic arterial reconstruction during LDLT and intraoperative doppler flowmeter assessment of liver allograft}

Hepatic arterial reconstruction was done by an endto-end anastomosis between graft and recipient hepatic arteriesusing "parachute technique" under surgical loupes. The detailed technique and its modificationsin presence of dual hepatic arteries is described earlier (1). In the recipients with poor caliber $\mathrm{HA}$, an extraanatomical reconstruction of the HA was done. In such situations, a supra-coeliac aorto-hepatic anastomosis using saphenous venous conduit was preferred technique for extra-hepatic source of graft hepatic arterial reconstruction (9). Immediately after the HA reconstruction, the arterial blood flow was measured by doppler flowmeter and any inadequacy was dealt. The anastomosis was repeated if any kinking, angulation or acute thrombosis of HA was noted intraoperatively. Doppler ultrasonography (USG)of liver was performed on postoperative day 1,3 , and 7 and then weekly thereafter for $1^{\text {st }}$ month to assess the hepatic arterial flow, portal venous flow, graft tissue perfusion, venous outflow, and graft regeneration.A resistive index (RI) of 0.4 or less on doppler study was considered significant which was clinically correlated. In case of abnormal findings on doppler USG such as modification of systolic waveform, abnormal values for resistive index, and altered systolic acceleration time, in presence of clinical symptoms and sharply elevated liver enzymes, abdominal CT scan, angiography, or both were performed. 


\section{Immunosuppressive protocol}

The immunosuppressive protocol remained standard and described elsewhere (10). All the recipients received basiliximab (simulect) $20 \mathrm{mg}$ intravenously at day 0 (within 6 hours of reperfusion of liver graft) and day 4 after transplantation. After $1 \mathrm{gm}$ of bolus of methylprednisolone intraoperatively after reperfusion of graft, steroids were tapered as per the standard protocol. We completely stop steroids after 2 weeks of initial administration and immunosuppressive regimen after 2 weeks in this case study primarily consisted of tacrolimus, mycophenolate mofetil (MMF) and everolimus. We included everolimus in our immunosuppressive protocol since February 2012 and continue to use it starting from early postoperative period after LDLT. Initial dose of everolimus is $0.25 \mathrm{mg} q 12$ hours and is increased to $0.5 \mathrm{mg}$ q12 hours to achieve a target trough level of $3-5 \mathrm{ng} / \mathrm{ml}$.

\section{Endovascular thrombolytic therapy by urokinase infusion}

The protocol of treating the HAT patients has been described earlier (1). Intra-arterial urokinase therapy was given whenever feasible and remained as first line of treatment for the patients with HAT that developed after 24 hours without clinical deterioration. First, a diagnostic CT arteriography was performed with standard catheter techniques using the right femoral arterial access with selective catheterization of the celiac trunk. Once HAT was confirmed, a micro catheter was manipulated into the thrombus. Thrombolysis was initiated with a bolus dose of 150,000 IU urokinase followed by continuous infusion. For continuous infusion, 1.5 Million IU of urokinase was added to $250 \mathrm{ml}$ of normal saline and was administered at the rate of $10 \mathrm{ml} /$ hour $(60,000 \mathrm{IU}$ of urokinase per hour) through a HA catheter. All patients were monitored in intensive care unit for any hemodynamic instability while on urokinase therapy. No heparin was given as a treatment or prophylaxis. The endovascular thrombolytic therapy was monitored using Doppler USG to ensure hepatic arterial canalization. Recanalization of the HA was assessed by presence of an arterial flow at the graft hilum as well as in the intrahepatic arterial branches which was confirmed by hepatic arteriography. The microcatheter was placed in situ for next 24 hours even after re-canalization.

Surgical exploration for thrombectomyand/or revision of $\mathrm{HA}$ anastomosis were considered if $\mathrm{HA}$ complications were detected in immediate postoperative period (within 24 hours) and, also, if urokinase infusion therapy failed.

\section{RESULTS}

A total of 908 recipients underwent LDLT (Male: Female, 665:243) with a mean age of 53 years (range, 1-76 years). 12 episodes of HAT (1.3\%) were diagnosed in postoperative period and managed as shown in fig. 1. No significant correlation was found between
Figure 1 - Outcome of the HAT patients after urokinase therapy

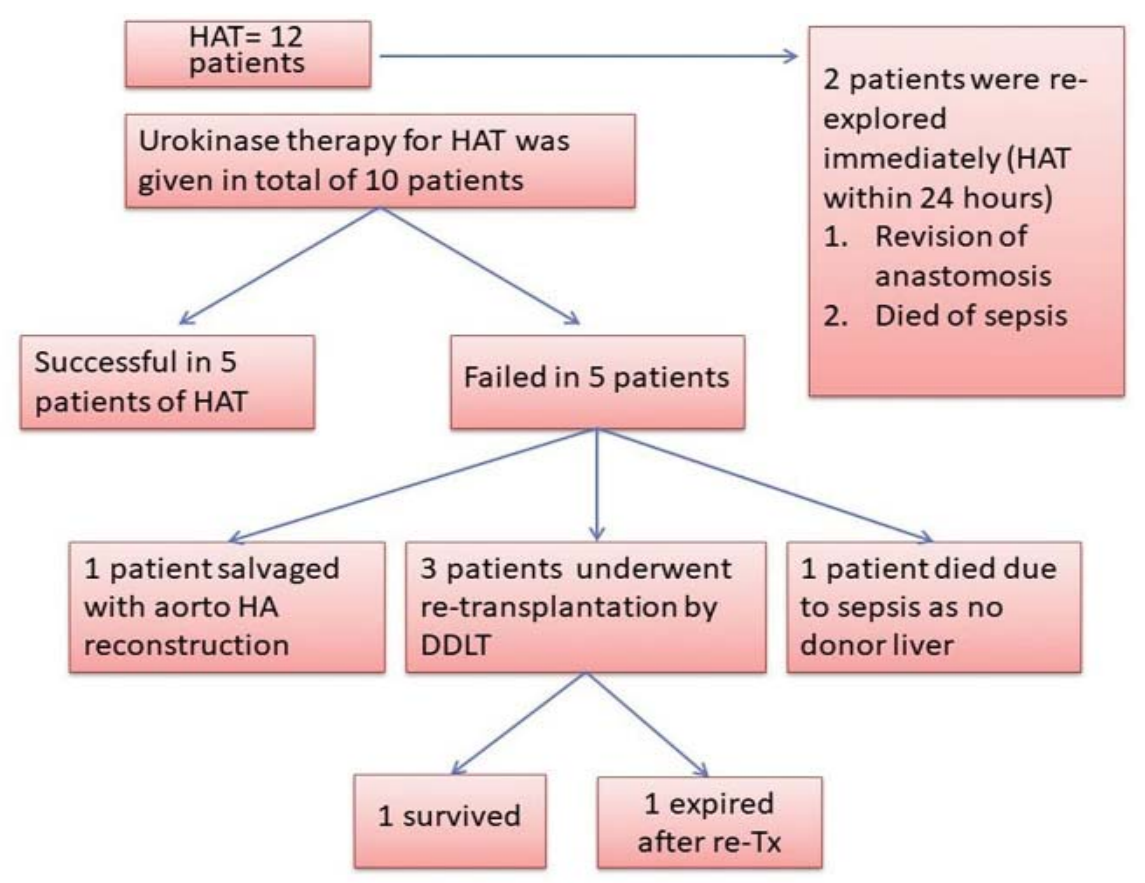


everolimus immunosuppression and occurrence of HAT. Two patients were diagnosed HAT within 24 hours after surgery. Eight patients developed HAT between 1 to 21 days after LDLT whereas two patients in this series had late HAT occurring in 2nd postoperative month.

Initial surgical revascularization was attempted in two patients in immediate postoperative without administering the urokinase therapy due to acute HAT and worsening clinical condition. Anastomosis was revised successfully in one patient whereas in another patient revision of hepatic arterial anastomosiswas not possible due to intimal dissection of the graft HA. The patient died of sepsis while he was waitlisted for urgent deceased donor liver transplantation (DDLT).

Remaining ten patients who were diagnosed to have HAT after 24 hours of LDLT were treated with intraarterial urokinase infusion. Five of these patients (41.6\%) showed complete recanalization of the HA after 24 hours of thrombolytic therapy without any liver function abnormalities (fig. 2). All the patients were monitored in an intensive care unit for hemo-dynamic disturbances. None of the patient developed hemorrhagic episodes secondary to thrombolytic therapy.

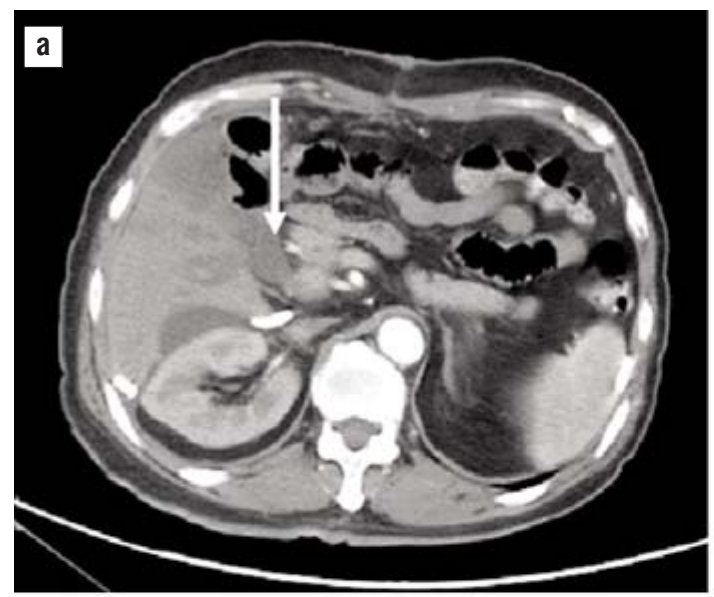

Figure 2 - a) CT angiography showing lack of flow in HA. b) Coeliac arteriography showing abrupt end of hepatic arterial flow suggestive of HAT. c) Establishment of hepatic arterial flow after urokinase therapy
Urokinase treatment was not effective in five patients. Three patients were re-explored, and hepatic arterial anastomosis revision was attempted (table 1). Due to intimal dissection of recipient HA, a supracoeliac aorto-hepatic conduit was used to establish arterial flow in one patient where has two patients underwent emergency DDLT as revision of anastomosis was not possible. One patient died of sepsis before any intervention was attempted. One patient developed HAT on 8th day post-LDLT. Urokinase therapy was administered, but re-canalization could not be achieved. This patient was salvaged by an emergency DDLT who continues to do well till latest follow up with stable liver functions. Among the HAT patients $(n=12)$, the mortality directly related to thrombosis leading to graft failure was $25 \%$ ( $n=3)$.

There was no rebound HAT in the surviving patients with no evidence of acute cellular rejection episodes.

\section{DISCUSSION}

HAT is most common HA related complications occurring in post-transplant period after LDLT that can
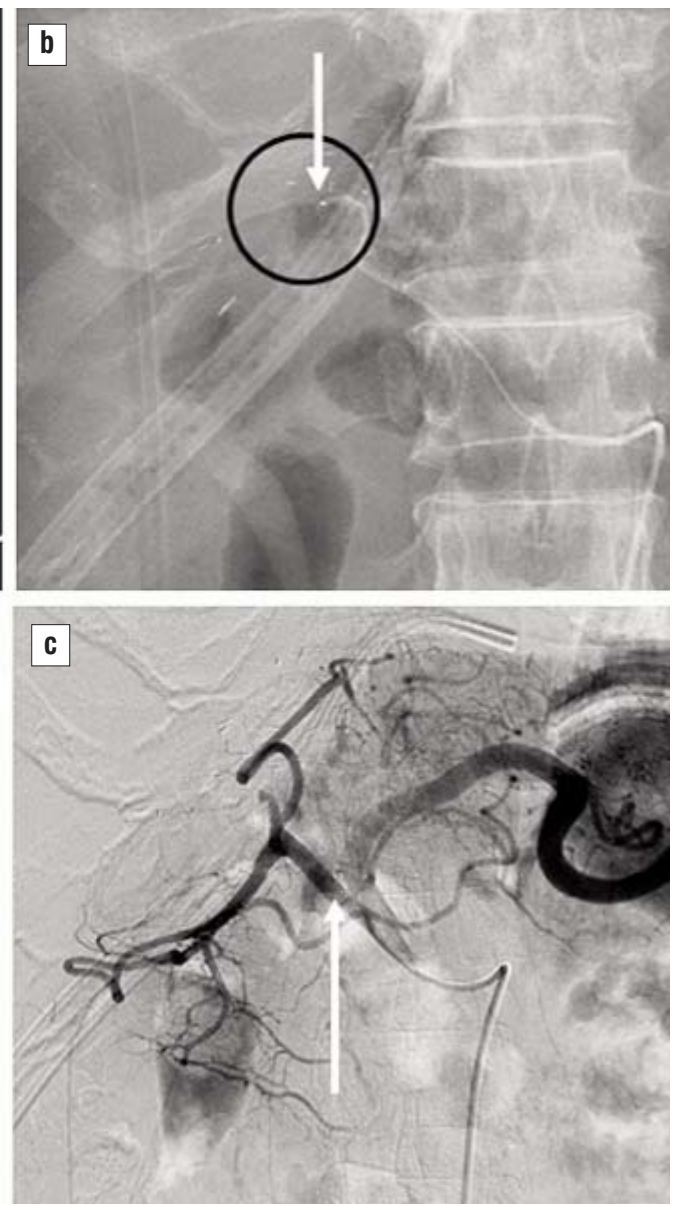
Table 1 - Treatment modalities in recipients that required surgical intervention(s) due to failure of urokinase therapy

\begin{tabular}{|c|c|c|c|c|c|}
\hline & Type of graft & Urokinase therapy & Surgical revascularization & Re-Transplantation & Status \\
\hline Recipient 1 & Right lobe LDLT & $\begin{array}{l}\text { Yes } \\
\text { Not successful }\end{array}$ & $\begin{array}{l}\text { Extra-anatomical hepatic arterial } \\
\text { reconstruction by supra-coeliac } \\
\text { aorto-hepatic conduit }\end{array}$ & - & Alive \\
\hline Recipient 2 & Right lobe LDLT & $\begin{array}{l}\text { Yes } \\
\text { Not successful }\end{array}$ & $\begin{array}{l}\text { Revision of anastomosis attempted, } \\
\text { but failed }\end{array}$ & DDLT & Alive \\
\hline Recipient 3 & Right lobe LDLT & $\begin{array}{l}\text { Yes } \\
\text { Not successful }\end{array}$ & $\begin{array}{l}\text { Revision of anastomosis attempted, } \\
\text { but failed }\end{array}$ & DDLT & $\begin{array}{l}\text { Died of } \\
\text { sepsis }\end{array}$ \\
\hline Recipient 4 & Right lobe LDLT & $\begin{array}{l}\text { Yes } \\
\text { Not successful }\end{array}$ & Not possible & Not possible & $\begin{array}{l}\text { Died of } \\
\text { sepsis }\end{array}$ \\
\hline Recipient 5 & Right lobe LDLT & $\begin{array}{l}\text { Yes } \\
\text { Not successful }\end{array}$ & $\begin{array}{l}\text { Not possible due to clinical } \\
\text { deterioration }\end{array}$ & $\begin{array}{l}\text { Re-Transplantation } \\
\text { by DDLT }\end{array}$ & Alive \\
\hline
\end{tabular}

* Urokinase therapy was successful in five patients achieving complete re-canalization without any complications

lead to allograft ischemia, which carries a mortality rate as high as $55 \%$ (8). Therefore, early detection of HAT remains critical and warrants urgent intervention to avoid hepatic infarction. Endovascular treatment as initial approach has been tried with variable success recently in many transplant centers. In present retrospective analysis, HA patency after intra-arterial thrombolysis was restored in $41.6 \%$ of the recipients. However, acute HAT detected in immediate post-transplant period are best treated by emergency surgical revascularizationprocedure $(11,12)$. In absence of hepatic arterial flow and development of hepatic infarction, retransplantation remains only option to salvage the patient. In our experience, HAT was detected in $1.3 \%(12 / 908)$ of the recipients post-LDLT with a mortality rate of $25 \%(3 / 12)$ despite of prompt medical and surgical management. Initial surgical revascularization was done in 2 patients due to worsening clinical condition and rapidly deteriorating liver allograft functions. However, in patients with HAT with stable liver functions, we do not routinely consider surgical intervention. As endovascular therapeutic options are available, the need for urgent re-transplantation for treatment of HAT is not routinely recommended especially patients with stable liver graft functions. Only in the case of initial nonfunction or deteriorating liver graft functions after several therapeutic attempts re-transplantation should be considered.

Endovascular therapeutic options such as intraarterial thrombolysis, percutaneous transluminal angioplasty, and stent placement have shown encouraging results in recent years; however, it remains a controversial subject because of potential risk of hemorrhage and hemodynamic instability during the treatment. Singhal et al (12) reviewed role of thrombolytic therapy in 69 recipients that developed HAT after liver transplantation. In their analysis, intraarterial thrombolysis was successful in 47 out of 69 (68\%) patients on whom the therapy was attempted. We used urokinase therapy as a first line of treatment in patients of HAT with stable clinical condition. In present case series, urokinase infusion was started during the CT angiography procedure immediately after diagnosis was confirmed. Failure of recanalization of the HA with deterioration of graft liver function warrants surgical intervention. However, initial surgical revascularization is an individual decision depending upon the liver allograft functions, timing of thrombosis, and opinion of the surgeon who performed the anastomosis as reoperation in early transplant period has high risk of postoperative insufficiency (8).

Early HAT manifests clinically as fever, leukocytosis, sharp elevation of liver enzymes, or septic shock. Hence, timing of the diagnosis is crucial in managing HAT. Protocol doppler USG can detect HAT prior to development of clinical manifestations. Doppler USG is a proven noninvasive investigation for assessment of HA patency. The most common findings on USG are absence of arterial signals (sensitivity $92 \%$ ) or decreased RI of the HA that can be correlated clinically (13). In this study, all cases of HAT were initially detected by USG that was followed by an abdominal contrast-enhanced CT angiography. Conventional catheter arteriography of coeliac trunk through a femoral catheter is helpful in delineating the anatomical accuracy of the extent of HAT and interventional therapy can be contemplated rapidly during imaging. Thrombolysis remains only intervention for patients with extended HAT involving entire intra-hepatic 
arterial system (12). Failure to achieve arterial patency and, thus, adequate inflow to liver allograft warrants an emergency re-transplantation as surgical revision of anastomosis has no role in such situation. After initial successful experience by Hidalgo et al (14), thrombolytic management for HAT after transplantation has evolved and can be used for both early as well as late HAT. Thrombolysis is believed to be more effective in fresh clots because of high water content and relatively fibrin-poor matrix.

Urokinase and alteplase, both, have been used successfully as thrombolytic agents. Although their biochemical actions are different and there is no added advantage of one drug over the other, urokinase remains commonly preferred thrombolytic drug in most of the published literature (15). There is no consensus on the optimal technique for catheterdirected delivery of any thrombolytic agent as they have been successfully used as continuous infusion or bolus form. However, direct intra-arterial infusion at the site of thrombosis is used in most centers including our center. This procedure has several advantages such as small thrombolytic dose, high localized concentration, and little influence on systemic coagulation (16). Different dose regimens of thrombolytic drugs have been described with equal safety and efficacy. However, the lowest effective dosage and duration remains to be determined. Usually administered dose of alteplase is $1-3 \mathrm{mg}$ and that of urokinase is 50,000-250 000 IU (17). We used urokinase as the thrombolytic therapy in the dose 60,000 IU per hour and the infusion was continued till HA patency was confirmed by repeat angiography. Five patients in this series were successfully treated using urokinase therapy without any liver graft related complications and rebound thrombosis. Recanalization of HA could not be achieved in five patients that needed surgical exploration. Hepatic inflow was successfully revised in one patient by aorto-hepatic conduit (9) whereas retransplantation was needed in three patients and one patient died of overwhelming sepsis before any surgical intervention could be arranged.

The coagulation profile of patient should be carefully monitored during thrombolytic therapy in an intensive care unit. Duration of the therapy largely depends on the restoration of hepatic inflow and clinical parameters of the patient. If there is residual thrombus, deterioration of clinical status, or persistent HAT after 36 hours of continuous infusion, urokinase therapy should be stopped. Surgical intervention by revision of anastomosis or re-transplantation are the only available in such situation (8). Fatal intrabdominal hemorrhage can complicate the urokinase therapy and has been reported up-to $20 \%$ of the patients (12). In the present study cohort, there was no incidence of hemorrhagic complication secondary to urokinase infusion.

\section{CONCLUSION}

In conclusion, HAT is major HA related complication after LDLT and carries a high mortality risk if not treated promptly. Pulsed doppler USG of HA should be the initial imaging test for the patients suspected to have HAT that should be followed by CT angiography. The choice of treatment modality for HAT depends on the clinical status of the patient. Our successful experience with endovascular thrombolytic therapy with urokinase highlights the alternate approach to surgery and emergency re-exploration can be avoided in nearly $50 \%$ of HAT patients. Urokinase therapy should be carefully titrated and monitored. Failure of hepatic arterial recanalization or clinical deterioration of the patient warrants urgent surgical intervention either by revision of anastomosis or re-transplantation.

Financial support: no financial Funding from any source.

Conflicts of interest: no conflicts of interest.

\section{REFERENCES}

1. Li PC, Thorat A, Jeng LB, Yang HR, Li ML, Yeh CC, et al. Hepatic artery reconstruction in living donor liver transplantation using surgical loupes: Achieving low rate of hepatic arterial thrombosis in 741 consecutive recipients-tips and tricks to overcome the poor hepatic arterial flow. Liver Transpl. 2017;23(7):887-898.

2. Abdelaziz 0, Hosny K, Amin A, Emadeldin S, Uemoto S, Mostafa M. Endovascular management of early hepatic artery thrombosis after living donor liver transplantation. Transpl Int. 2012;25(8):847-56. doi: 10.1111/j.1432-2277.2012.01509.x. Epub 2012 Jun 18.

3. Scarinci A, Sainz-Barriga M, Berrevoet F, van den Bossche B, Colle I, Geerts $A$, et al. Early arterial revascularization after hepatic artery thrombosis may avoid graft loss and improve outcomes in adult liver transplantation. Transplant Proc. 2010;42(10):4403-8. doi: 10.1016/j.transproceed.2010.07.014.

4. Stange BJ, Glanemann M, Nuessler NC, Settmacher U, Steinmüller T, Neuhaus P. Hepatic artery thrombosis after adult liver transplantation. Liver Transpl. 2003;9(6):612-20.

5. Tanaka K, Uemoto S, Tokunaga Y, Fujita S, Sano K, Nishizawa T, et al. Surgical techniques and innovations in living related liver transplantation. Ann Surg. 1993;217(1):82-91.

6. Kamran Hejazi Kenari S1, Mirzakhani H, Eslami M, Saidi RF. Current state of the art in management of vascular complications after pediatric liver transplantation. Pediatr Transplant. 2015;19(1):18-26. doi: 10.1111/petr.12407. Epub 2014 Nov 25

7. Kim BW, Won JH, Lee BM, Ko BH, Wang HJ, Kim MW. Intraarterial thrombolytic treatment for hepatic artery thrombosis immediately after living donor liver transplantation. Transplant Proc. 2006;38(9): 3128-31.

8. Stange BJ, Glanemann M, Nuessler NC, Settmacher U, Steinmüller T, Neuhaus P. Hepatic artery thrombosis after adult 
liver transplantation. Liver Transpl. 2003;9(6):612-20.

9. Li PC, Thorat A, Jeng LB, Yang HR, Li ML, Yeh CC, et al. Successful application of supraceliac aortohepatic conduit using saphenous venous graft in right Lobe living donor liver transplantation. Liver Transpl. 2017;23(7):976-980. doi: 10.1002/lt.24720.

10. Jeng LB, Thorat A, Hsieh YW, Yang HR, Yeh CC, Chen TH, et al. Experience of using everolimus in the early stage of living donor liver transplantation. Transplant Proc. 2014;46(3):744-8. doi: 10.1016/j. transproceed.2013.11.068.

11. Mourad MM, Liossis C, Gunson BK, Mergental H, Isaac J, Muiesan $P$, et al. Etiology and management of hepatic artery thrombosis after adult liver transplantation. Liver Transpl. 2014;20(6):713-23. doi: 10.1002/lt.23874.

12. Singhal A, Stokes K, Sebastian A, Wright HI, Kohli V. Endovascular treatment of hepatic artery thrombosis following liver transplantation. Transpl Int. 2010;23(3):245-56. doi: 10.1111/j.1432-2277. 2009.01037.x. Epub 2009 Dec 22.

13. López-Benítez R, Schlieter M, Hallscheidt PJ, Radeleff BA Kauffmann G, Richter GM, et al. Successful arterial thrombolysis and percutaneous transluminal angioplasty for early hepatic artery thrombosis after split liver transplantation in a four-month-old baby. Pediatr Transplant. 2008;12(5):606-10. doi: 10.1111/j.1399-3046. 2008.00925.x.

14. Hidalgo EG, Abad J, Cantarero JM, Fernández R, Parga G, Jover JM, et al. High-dose intra-arterial urokinase for the treatment of hepatic artery thrombosis in liver transplantation. Hepatogastroenterology. 1989;36(6):529-32.

15. Semba CP, Bakal CW, Calis KA, Grubbs GE, Hunter DW, Matalon TA, et al. Alteplase as an alternative to urokinase. Advisory Panel on Catheter-Directed Thrombolytic Therapy. J Vasc Interv Radiol. 2000; 11(3):279-87.

16. Li ZW, Wang MQ, Zhou NX, Liu Z, Huang ZQ. Interventional treatment of acute hepatic artery occlusion after liver transplantation. Hepatobiliary Pancreat Dis Int. 2007;6(5):474-8.

17. Boyvat $F$, Aytekin C, Firat A, Harman A, Karakayali $H$, Haberal $M$. Diagnostic and therapeutic management of hepatic artery thrombosis and stenosis after orthotopic and heterotopic liver transplantation. Transplant Proc. 2003;35(7):2791-5. 